\title{
Duloxetine Exposure During Pregnancy and the Risk of Spontaneous and Elective Abortion: A Danish Nationwide Observational Study
}

\author{
Mikkel Zöllner Ankarfeldt ${ }^{1,7}$ (i) · Janne Petersen ${ }^{1,2}$. Jon Trærup Andersen ${ }^{3,4}$. \\ Maria Fernanda Scantamburlo Fernandes ${ }^{5} \cdot \mathrm{Hu} \mathrm{Li}^{5} \cdot$ Stephen Paul Motsko ${ }^{5}$. Thomas Fast ${ }^{6}$. Espen Jimenez-Solem ${ }^{1,3,4}$
}

Accepted: 22 April 2021 / Published online: 18 May 2021

(c) The Author(s) 2021

\begin{abstract}
Background Depression and antidepressant treatment are widespread among women of childbearing age.

Objective This study evaluates the association between duloxetine exposure during pregnancy and spontaneous and elective abortions.

Patients and Methods The nationwide, observational study based on register data from Denmark included women with a recorded pregnancy in the birth register or an abortion in the patient register between 2004 and 2016. Duloxetine-exposed women were compared with (1) duloxetine non-exposed, (2) selective serotonin reuptake inhibitor (SSRI)-exposed, (3) venlafaxine-exposed, and (4) women discontinuing duloxetine before pregnancy. Exposure status was based on records of redeemed prescriptions. Cox regression with adjustments and propensity score matching was applied.

Results The data from 1,019,957 pregnancies were used, including 1,212 pregnancies exposed to duloxetine. Duloxetineexposed women had an increased hazard ratio (HR) for spontaneous abortions compared with SSRI-exposed women: propensity score matched HR 1.25 [95\% confidence interval (CI), 1.00-1.57]. No increased hazard was observed for duloxetine-exposed women compared with duloxetine non-exposed: 1.08 (95\% CI 0.89-1.31); venlafaxine-exposed: 1.08 (95\% CI 0.82-1.41); and duloxetine discontinuers: 0.99 (95\% CI 0.76-1.30). An increased HR of elective abortions was observed in duloxetine-exposed women compared to duloxetine non-exposed: 1.41 (95\% CI 1.25-1.59); SSRI-exposed: 1.32 (95\% CI 1.15-1.51); and duloxetine discontinuers: 1.46 (95\% CI 1.23-1.75), but not to venlafaxine-exposed women: 1.09 (95\% CI 0.93-1.27).

Conclusion There was no increased risk of spontaneous or elective abortion associated with exposure to duloxetine. The increase risk observed for women exposed to duloxetine in comparison with SSRI-exposed for spontaneous and in comparison with all groups (except venlafaxine-exposed) for elective abortion suggested confounding.
\end{abstract}

\section{Key Points}

The risk of elective and spontaneous abortion after use of duloxetine during pregnancy was investigated.

Patients that use duloxetine during pregnancy may have an increased risk of spontaneous or elective abortion due to for example health behavior compared to other pregnant women. It is difficult to investigate the risk of duloxetine alone.

There was no increased risk of spontaneous or elective abortion associated with exposure to duloxetine.

Mikkel Zöllner Ankarfeldt

Mikkel.Zoellner.Ankarfeldt@ regionh.dk;

mikkelza@gmail.com

Extended author information available on the last page of the article

\section{Introduction}

The prevalence of both depressive symptoms and major depression is high among pregnant women, affecting up to $15 \%$ [1-4] and $10 \%$ [5], respectively. Antidepressants are a pharmacological treatment option and up to $13 \%$ [6-9] of pregnant women are treated. The most common antidepressants are selective serotonin reuptake inhibitors (SSRIs) [9-12] and the second most common are serotonin and norepinephrine reuptake inhibitors (SNRIs), including duloxetine and venlafaxine [9, 10, 13]. In Denmark, SSRIs are a first-line treatment, whereas SNRIs are a secondline treatment [14]. Duloxetine is indicated for the treatment of major depressive disorders, generalized anxiety disorders, stress urinary incontinence, diabetic peripheral neuropathic pain, and, in some countries (e.g., in the USA) for fibromyalgia. 
Bassiouni et al. [15] found in 1979 that the blood concentration of serotonin was higher in women with spontaneous abortions than in those who have given birth. Since then, SSRIs and other antidepressants affecting the serotonergic system (e.g., SNRIs) have been suspected of causing negative birth outcomes. However, subsequent studies investigating miscarriage among pregnant women treated with SSRIs have shown contradictory results [16-24]. Women with a diagnosis of a major depressive disorder are more likely to smoke or use alcohol or other substances, which may confound the study results $[25,26]$. It remains unclear whether the associations between SSRIs and spontaneous or elective abortions are due to biological or behavioral factors intrinsic to women with mood disorders, to medications used to treat the disorder, or a combination of both. Available data regarding the safety of SNRIs in relation to spontaneous or elective abortion are sparse. A UK-based study [27] investigating 281 pregnant women exposed to the SNRI venlafaxine found a non-significant association with spontaneous abortions when compared with those who were not exposed to antidepressants: hazard ratio (HR) 1.3 [95\% confidence interval (CI), 0.9-1.9], and no association when compared with SSRI-exposed pregnant women: HR 1.0 (95\% CI 0.7-1.6), adjusting for history of abortions and duration of pregnancy. A Canada based study [28] found an increased prevalence of elective abortion among women exposed to antidepressants, and for women exposed to SNRIs, an increased risk of spontaneous abortion when accounting elective abortion, compared to unexposed women with and without depression. A Scandinavian case-control study [29] investigated elective abortions and found that the use of any type of antidepressants, or specifically venlafaxine, was positively associated with elective abortions compared to non-exposed pregnancies: odds ratio 1.7 (95\% CI 1.5-1.8) and 1.6 (95\% CI 1.4-1.7). When restricting to only elective abortions due to a malformation, no association was observed. A Danish study [23] found a positive association between duloxetine and spontaneous abortions; however, the analyses were unadjusted, the cohort covered pregnancies from 1997 to 2008, and the findings were attributed to confounding. While an increased risk of spontaneous abortion can be a safety problem, an increased risk of elective abortion is more challenging to interpret. The decision of an elective abortion is likely multifactorial [30], but may be due to a fetus malformation diagnosis. To improve knowledge regarding the safety of duloxetine during pregnancy, the present study evaluates the association between maternal exposure to duloxetine and spontaneous or elective abortions. Four prespecified comparator groups were used: duloxetine non-exposed, SSRI-exposed, venlafaxineexposed, and duloxetine discontinuers.

\section{Methods}

The present observational study used data from the Danish National Prescription Register [31, 32], the Danish National Patient Register [33], the Medical Birth Register [34, 35], and registers containing information on education and household income [36, 37]. In Denmark, elective abortion is legal and freely available until the end of gestational week 12. After week 12, elective abortion can still be performed if, among others, the woman's somatic or mental health would deteriorate due to the pregnancy, she is incapable of providing proper care to the child due to her somatic or mental health status, or there is a risk of serious birth defects for the fetus [38, 39].

The Danish national registers are protected by the Danish Act on Processing of Personal Data and can only be accessed following an approved application. The Danish Data Protection Agency approved the study (j.nr. VD-2018-371, I-Suite nr. 6621). Due to the study's observational design, no ethics committee approval was needed. Personal identification numbers were encrypted for the researchers.

\subsection{Participants}

All pregnancies in Denmark with a hospital contact encounter from 2004 to 2016 were eligible for the study population. The Medical Birth Register, which has a completeness of $99 \%$, provided information on all live births and stillbirths [40-43]. Data on pregnancies ending with an abortion diagnosis (elective or spontaneous) were gathered from the National Patient Register. Gestational age for live births and abortions was identified in the medical birth register and the patient register, respectively. Information on early abortions with no hospital contact was not available. Mothers migrating 12 months prior to their last menstrual period (LMP) until 1 month after giving birth were excluded to ensure that the women were in the Danish healthcare system and their data were available in the registers.

\subsection{Exposure, Comparison Groups, Outcomes, and Covariates}

Information on maternal exposure to antidepressant drugs was based on redeemed prescriptions from community pharmacies using the Danish National Prescription Register. Exposure during pregnancy was determined as redemption of a prescription in the time window from 30 days prior to LMP to 140 days post LMP, or the end of the pregnancy, whichever came first. Women exposed to duloxetine (ATC N06AX21) were compared with four prespecified comparison groups: (1) Duloxetine non-exposed: no redeemed prescriptions of duloxetine in the exposure time window, to 
have a broad, diverse comparison group. (2) SSRI-exposed: a redeemed prescription of an SSRI (ATC N06AB) and no redeemed prescription of duloxetine, to have a comparison group with exposure to antidepressants, but in another drug class than duloxetine. (3) Venlafaxine-exposed: a redeemed prescription of venlafaxine (ATC N06AX16) and no redeemed prescription of duloxetine, to have a comparison group with exposure to an antidepressant in the same drug class as duloxetine and therefore a comparable indication for the treatment (e.g., depression severity). Women with duloxetine exposure from 90 days to 30 days prior to LMP were excluded from the duloxetine non-exposed, SSRI-exposed, and venlafaxine-exposed comparison groups to ensure no duloxetine exposure in the exposure time window. (4) Duloxetine discontinuers: at least one redeemed prescription of duloxetine between 365 days prior to LMP to 30 days prior to LMP, but no redeemed prescription of duloxetine in the exposure time window, to have a comparison group of women known to recently have an indication for duloxetine. The comparison groups were not mutually exclusive and were analyzed separately.

Information on the abortion outcomes (ICD-10 codes for spontaneous abortions: O021 and O03; elective abortions: O04, O05, and O06) were collected from the Danish National Patient Register, which holds data on all procedures and diagnoses relating to hospital contact. The reasons for elective abortion were not available in the register.

Based on literature, clinical knowledge, and available data in the registers, the following possible confounders were prespecified for adjustment in the logistic regression and for the propensity score (PS) model: birth year of the offspring (three categories: 2004-2008, 2009-2012, 2013-2016), maternal age (four categories: 18-24, 25-29, $30-34,>34$ years), psychiatric hospitalizations (1 year prior to LMP: yes/no), psychiatric outpatient visits (1 year prior to LMP: yes/no), household income (year of LMP, grouped in quartiles), and education (highest completed education in the year of LMP, three categories: $<11,11-15$, and $>15$ years). Co-morbidities 5 years prior to LMP (see Table S1 in the Online Supplementary Material (OSM) for ICD-10 codes and ATC codes) included: affective disorder, anxiety or phobia, depression, diabetes during pregnancy, diabetes, diabetic peripheral neuropathic, hyper- and hypothyroidism, hypertension, obesity, renal failure, severe stress reaction, and stress urinary incontinence. Co-medication of prescriptions redeemed 90 days prior to LMP to 140 days post LMP, or the end of the pregnancy, whichever came first (see Table S2 in the OSM for ATC codes) included: antiepileptics, antihypertensives, antipsychotics, anxiolytics, danazol, estradiol, fluconazole, glucose-lowering drugs, non-steroidal anti-inflammatory drugs, opioids, progesterone, steroid hormones, and thyroid hormones.
Missing values in income were imputed 1 year prior to LMP if missing at the year of LMP and imputed 1 year after LMP if still missing. If education was missing at the year of LMP, imputation 1 year after LMP was done. After imputation, data were analyzed assuming missing at random, meaning that patients were deleted from the analyses if they still had missing values. There were $1.5 \%$ missing values for household income and $1.6 \%$ for education after imputation.

\subsection{Statistical Methods}

Separate Cox proportional hazard analyses were conducted to compare exposure to duloxetine with the four comparator groups individually. In the analyses with spontaneous abortions as the outcome, the risk time was censored at the event of elective abortion, and vice versa. In the analyses of spontaneous abortions, time was censored at week 22 , as abortions hereafter are defined as stillbirths. In the analyses of elective abortions, observation time was throughout the pregnancy. For each of the four comparator groups, an unadjusted, an adjusted, and a PS-matched analysis was performed. When fitting the adjusted model or PS model for each outcome and comparison group, all prespecified co-variates were included. Covariates where removed if parameter coefficients were not identifiable due to too much collinearity or too few observations. The final model for each analysis can be found in Table S4 (ESM).

Logistic regression was used to estimate PS. Greedy matching was performed with the SAS macro OneToMan$y M T C H$ [44] with an extension to match only women with a maximum of 0.2 logit (PS) difference. Duloxetine-exposed and duloxetine non-exposed women were matched with a 1:4 ratio. Duloxetine-exposed and SSRI-exposed women were matched with a 1:2 ratio, while duloxetine-exposed women were matched with venlafaxine-exposed women and duloxetine discontinuers with a 1:1 ratio, due to a differing number of women in the other comparison groups. Few duloxetine-exposed women were not matched (ranging between $1.2 \%$ and $8.4 \%$ depending on comparator; the numbers of women matched are shown in figures presenting results from the analyses) and were not included in the PSmatched analyses. In the PS-matched sample, a Cox proportional hazard regression stratified on matching group ID was used. The balance of possible confounders after PS matching was assessed with standardized mean differences using the SAS macro stddiff macro $[45,46]$.

Three prespecified sensitivity analyses were performed: (1) Exposure to duloxetine and comparators was redefined to a minimum of two redeemed prescriptions within the exposure time window, resulting in fewer exposed women. (2) Exposure to duloxetine and comparators was redefined to overlap between the exposure time window and days' supply of redeemed pills. This resulted in a higher number of 
exposed women since women with prescriptions redeemed just prior to the exposure time window were also defined as exposed. Days' supply was calculated based on the number of redeemed pills and their strength compared to the World Health Organization's defined daily dose [47]. (3) Events during a woman's first pregnancy may influence later pregnancies; therefore, additional sensitivity analyses were performed, restricting the cohorts to the first pregnancy occurring within the study period.

Describing the duration of the pregnancy/gestational age ending in elective abortion for each comparison group was decided as a post hoc analysis.

All analyses were performed using SAS Enterprise Guide 7.15 , and a significance level of $5 \%$ was applied. Validation of the programming was performed by an independent statistical programmer. The present study is based on a safety study regarding duloxetine and pregnancy outcomes, with the protocol and the full study report available via the European Network of Centres for Pharmacoepidemiology and Pharmacovigilance (ENCePP, EUPAS20253).

\section{Results}

The final cohorts for the analyses included 1,020,288 pregnancies, of which 1212 were duloxetine-exposed (Fig. 1). Baseline characteristics before and after PS-matching are shown in Table 1. Characteristics of duloxetine-exposed women were most comparable to venlafaxine-exposed and duloxetine discontinuers and least comparable to duloxetine non-exposed before PS-matching. After PS-matching, the distribution of the co-variates was balanced across comparators. SSRI co-medication was most unbalanced for the venlafaxine comparison group. Co-morbidity diagnoses were only available from hospital contacts and the proportion of pregnancies with co-morbidities may therefore be underestimated. Table S3 (OSM) shows the number of events, number of exposed, and crude prevalence rates (events per thousand pregnancies).

\subsection{Comparative Analyses of Spontaneous Abortion}

Figure 2 shows the results of the analyses of spontaneous abortions. When duloxetine-exposed women were compared with duloxetine non-exposed, a statistically nonsignificant increased hazard of spontaneous abortions was seen in the adjusted and PS-matched analyses: HR 1.14 (95\% CI 0.96-1.34) and 1.08 (95\% CI 0.89-1.31), respectively. A similar result was seen when comparing duloxetine with venlafaxine-exposed women: adjusted HR 1.18 (95\% CI 0.98-1.42); PS-matched 1.08 (95\% CI 0.82-1.41). No increase in hazard of spontaneous abortions was observed when duloxetine-exposed women were compared with duloxetine discontinuers: adjusted HR 1.04 (95\% CI 0.83-1.30); PS-matched 0.99 (95\% CI 0.76-1.30). When compared with SSRI-exposed, a statistically significant increased hazard of spontaneous abortions was observed for duloxetine-exposed women, with HR 1.23 (95\% CI $1.02-1.48)$ for the adjusted analysis and 1.25 (95\% CI $1.00-1.57)$ for the PS-matched analysis.

The sensitivity analyses defining exposure as more than one redeemed prescription and restricted to the first observed pregnancy showed a similar tendency as the main analyses, although with increased point estimates and wider
Fig. 1 Flow chart. LMP last menstrual period, SSRI selective serotonin reuptake inhibitor
Pregnancies of women with valid personal identification number, ending in birth, stillbirth, or abortion (elective or spontaneous) between 2004-2016 $\mathrm{N}=1,059,934$

Mother emigrated from 12 months prior to LMP until 1 month post end-of-pregnancy $\mathrm{N}=39,646$

Redeemed prescription of duloxetine 3 months prior to LMP and not during exposure period $\mathrm{N}=331$

Total pregnancies $\mathrm{N}=1,019,957$

- Duloxetine: 1,212

- Duloxetine non-exposed: $1,018,745$

- SSRI: 28,345

- Venlafaxine: 4,908

LMP: last menstrual period. SSRI: selective serotonin reuptake inhibitor. 


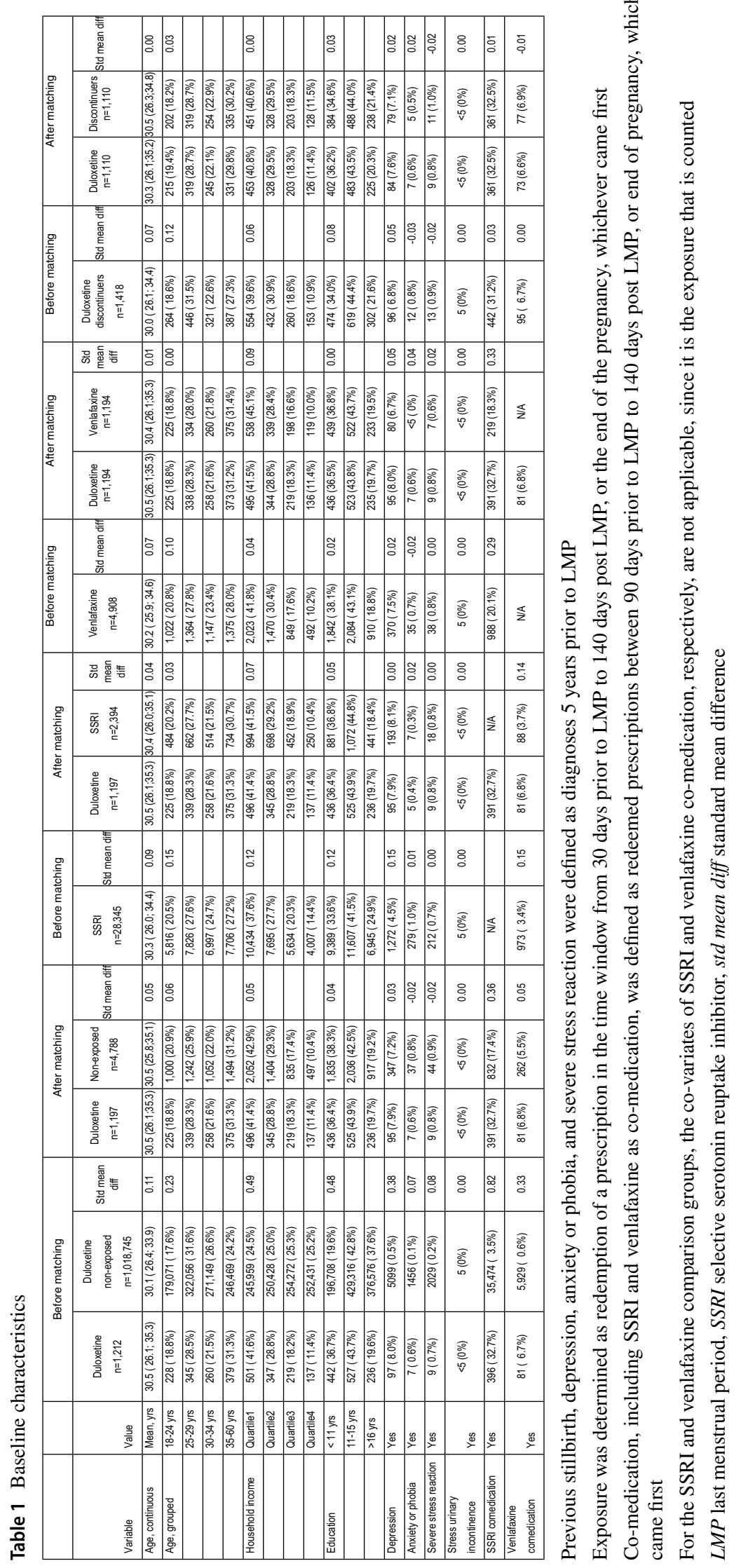


confidence intervals. In the sensitivity analyses defining exposure as overlap between days' supply and exposure window, smaller point estimates and wider confidence intervals were seen. See Figs. S1-S3 in the OSM.

\subsection{Comparative Analyses of Elective Abortion}

Figure 3 shows the analyses of elective abortions. Duloxetine-exposed women had an increased hazard for elective abortions when compared with duloxetine non-exposed, SSRI-exposed, and duloxetine discontinuers, respectively. For duloxetine-exposed compared with duloxetine nonexposed: the adjusted HR was 1.45 (95\% CI 1.31-1.60); PS-matched HR was 1.41 (95\% CI 1.25-1.59). When comparing duloxetine-exposed with SSRI-exposed, the adjusted HR was 1.35 (95\% CI 1.22-1.50); PS-matched HR was 1.32 (95\% CI 1.15-1.51). When comparing duloxetine-exposed with duloxetine discontinuers, the adjusted HR was 1.41 (95\% CI 1.21-1.64); PS-matched HR was 1.46 (95\% CI 1.23-1.75). A statistically non-significant increased hazard of elective abortions was observed when comparing duloxetine-exposed women with venlafaxine-exposed: adjusted HR 1.10 (95\% CI 0.98-1.23); PS-matched HR 1.09 (95\% CI 0.93-1.27).

In the sensitivity analyses defining exposure as more than one redeemed prescription, increased HRs with narrower CIs were seen. In the remaining sensitivity analyses, similar findings to the main analyses were observed. See Figs. S4-S6 in the OSM.

In the post hoc analysis, the median duration of pregnancies ending in elective abortions was 56 days for duloxetine-exposed, duloxetine non-exposed, SSRI-exposed, and venlafaxine-exposed women, and 55 days for duloxetine discontinuers. The 95 th percentiles ranged between 80 days (for duloxetine-exposed) and 88 days (for duloxetine nonexposed women).

\section{Discussion}

The present observational study, based on nationwide data registers from Denmark, investigated the association between duloxetine exposure during pregnancy and spontaneous and elective abortions. For spontaneous abortions, the analysis showed no statistically significant association when comparing duloxetine-exposed women with duloxetine non-exposed, venlafaxine-exposed, or duloxetine discontinuers. However, a statistically significant increased hazard was found when comparing duloxetine-exposed women with SSRI-exposed women. For elective abortions, both the PS-matched and adjusted analyses pointed towards an increased hazard of around $40 \%$ for women exposed to duloxetine across comparators, except when compared with venlafaxine, where no difference in hazard was found. Duration of pregnancies ending in elective abortion was similar across comparison groups.

\subsection{Interpretation}

The present study is based on valid information from established registries and includes a nationwide population of pregnant women with detailed information about antidepressant exposure, abortion outcome, and a range of important potential confounders covering co-medication, co-morbidity, and socioeconomic factors. Overall, the point estimates were greatly reduced in the adjusted and PS-matched analyses compared to the unadjusted analyses, indicating that confounding is present in the unadjusted results. Studies have shown that women with a depressive disorder are more vulnerable and are likely to have poorer health behavior during pregnancy (including smoking and use of alcohol) than women without depression [24-26]. This may confound the association between duloxetine and pregnancy outcomes when compared to the duloxetine non-exposed group, where most women are without depression and antidepressant medication. In the present study, vulnerability and health behavior were indirectly accounted for via, for example, education and income; however, information about alcohol intake, smoking, physical activity, illicit drug use, and folic acid supplementation during pregnancy was not available. Depression severity is another important confounder. The present study did strive to take depression severity into account by including information about depression diagnosis, the number of psychiatric hospital visits and psychiatric outpatient visits, although these are only proxies. Information about depression diagnosis was only available if the patients were admitted to a hospital. Consequently, it was not possible to identify all women suffering from depression, although we believe that women with the most severe depression were identified. However, some residual confounding is possible. In addition, individual women's indication for duloxetine was not available. Since duloxetine is indicated to treat depression, but also anxiety, stress urinary incontinence, and diabetic peripheral neuropathic pain, confounding by indication may affect the findings in the present study. We believe that the prevalence of these other indication diagnoses is low among women of childbearing age, while depression and anxiety are more prevalent. Nonetheless, the present study included co-morbidity of anxiety, diabetic peripheral neuropathic and stress urinary incontinence, registered in relation to hospital admissions, as co-variates. Finally, in order to compare duloxetine-exposed women with a group of women with similar characteristics and account for some of the unmeasured confounders, the present study also used women exposed to venlafaxine (a same-class medication) and duloxetine discontinuers as comparators. These 


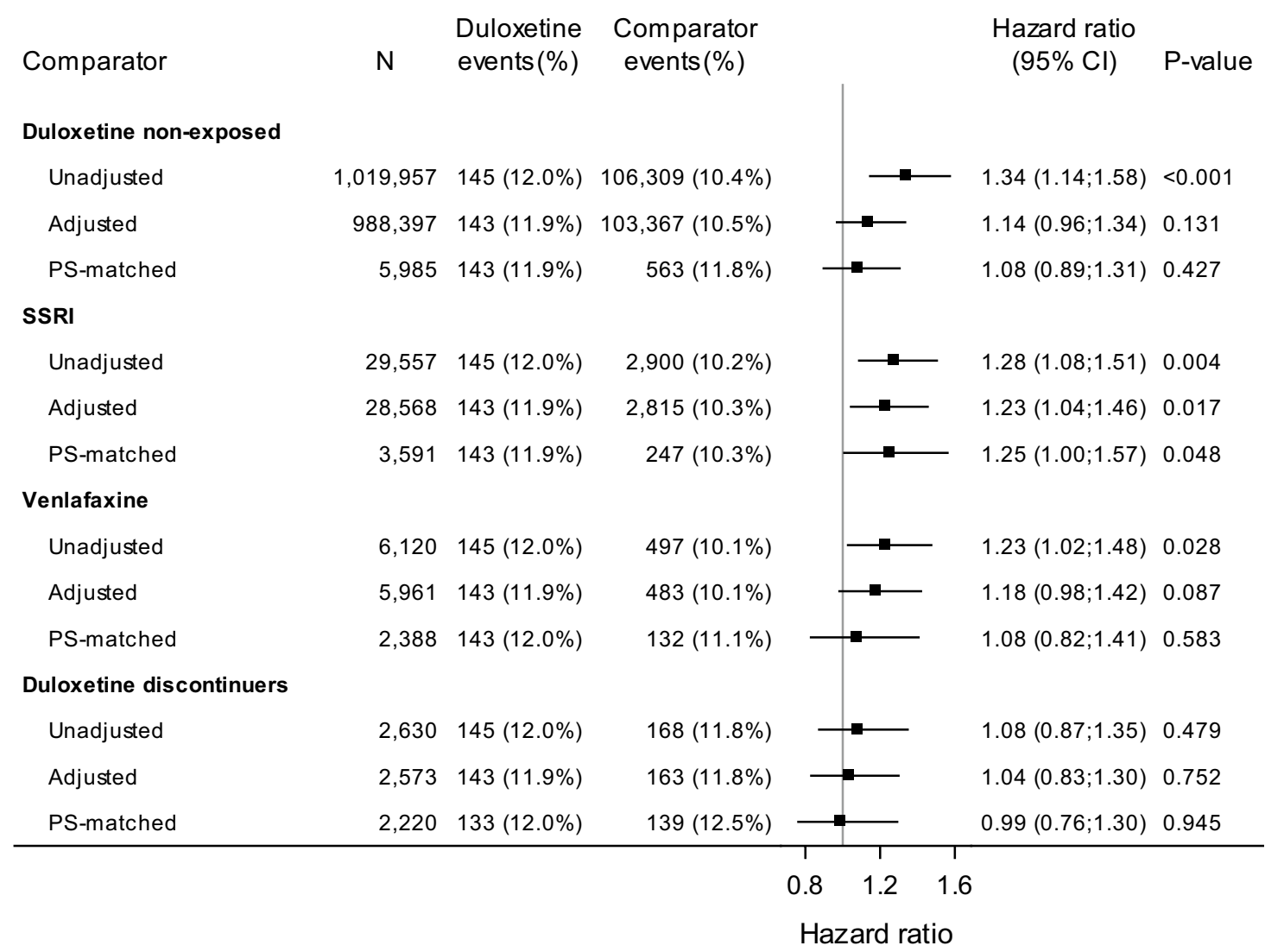

Fig. 2 Hazard ratios of spontaneous abortion: Duloxetine versus four comparators. Exposure definition: $\geq 1$ redeemed prescription; $\mathrm{N}$ : number of observations in analyses; CI: Wald 95\% confidence intervals; PS-matched: propensity score-matched analyses based on con- ditional logistic regression. Adjusted and PS-matched models were based on co-variates covering co-morbidity, co-medication, hospital contacts, education, and income. For the complete list for the individual analyses, see Online Supplementary Material Table S4 two groups are likely to have characteristics (including health behavior, depression severity, and drug indication) more comparable to duloxetine-exposed women than the comparator groups of duloxetine non-exposed and SSRIexposed women. In Denmark, both duloxetine and venlafaxine are often used for more severe depression, rather than SSRIs [14]. On the other hand, a comparison of duloxetine and venlafaxine may mask a drug class effect (SNRIs), and therefore lack of association cannot be interpreted alone.

Overall, duloxetine does not seem to increase the risk of spontaneous abortion. Although the analyses comparing duloxetine with SSRI-exposed women found an increased hazard, the results were not replicated in the analyses with other comparators. In the comparison with SSRI-exposed women, unmeasured confounding cannot be ruled out and the increased hazard could be explained by factors related to the exposure (e.g., confounding by depression severity), supported by the finding of no difference in hazard when duloxetine-exposed women were compared with duloxetine discontinuers, and a non-significant difference in hazard when duloxetine-exposed women were compared with venlafaxine-exposed women. A recent case-control study [48] based on 57,770 women from gynecological practices in Germany reported a positive association of spontaneous abortions for women with psychiatric disorders (e.g., depression, anxiety, adjustment disorder, somatoform disorder); however, it is a challenge to discern between possible risks related to drug exposure and risks related to underlying morbidity, especially when the results change across comparator groups and sensitivity analyses, as in the present study.

The sensitivity analyses of spontaneous abortion confirmed the findings of the main analyses, although results varied slightly depending on the definition of exposure and choice of cohorts. The redefinition of exposure as a minimum of two redeemed prescriptions aimed to increase the likelihood that the medication was consumed during pregnancy. The analyses showed a stronger statistically significant association when comparing duloxetine with SSRI and 


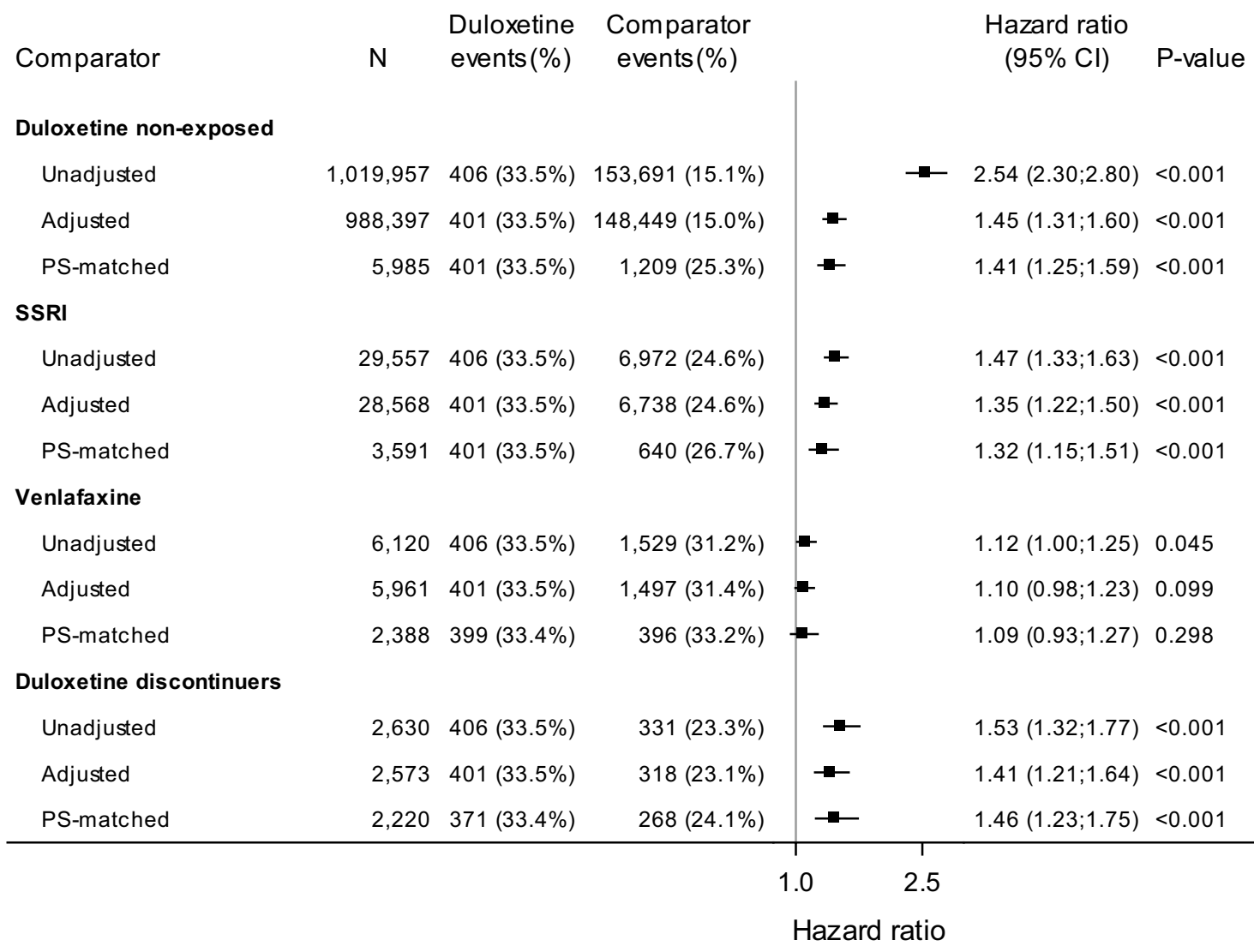

Fig. 3 Hazard ratios of elective abortion: duloxetine versus four comparators. Exposure definition: $\geq 1$ redeemed prescription; N: number of observations in analyses; CI: Wald 95\% confidence intervals; PSmatched: propensity score-matched analyses based on conditional

venlafaxine exposure. However, there was no increased hazard when duloxetine-exposed women were compared with duloxetine non-exposed women or with duloxetine discontinuers. Since a similar hazard was seen among duloxetineexposed women and discontinuers, an interpretation is that the increased risk observed in spontaneous abortions may be attributable to common characteristics, for example, health behavior such as illicit drug use and smoking. If this is the case, unmeasured confounding might be responsible for the increased hazard found when comparing duloxetine-exposed women with SSRI- and venlafaxine-exposed women. In the sensitivity analyses defining exposure on days' supply, associations with spontaneous abortions decreased or vanished across comparator groups. The reason for not observing an association could be the increased number of misclassified exposed subjects (women who redeem prescriptions prior to the exposure time window but do not take the medication during pregnancy) in these sensitivity analyses. With more misclassified exposed subjects, an association between drug and outcome may be attenuated. In the sensitivity logistic regression. Adjusted and PS-matched models were based on co-variates covering co-morbidity, co-medication, hospital contacts, education, and income. For the complete list for the individual analyses, see Online Supplementary Material Table S4

analyses of spontaneous abortions limiting the cohort to the first observed pregnancy, increased hazards of approximately $40 \%$ were observed across all comparator groups, although some were statistically non-significant. In general, primipara women tend to have greater pregnancy-specific distress and risk of spontaneous abortion than multipara women [49, 50]. This distress might be more pronounced for women exposed to duloxetine, attributable to unmeasured factors that increase the hazard of spontaneous abortions, such as alcohol intake [51], smoking [52], or other poor health behavior related to pregnancy, such as non-compliance with folic acid supplementation during pregnancy [53].

The number of studies analyzing duloxetine and the hazard of spontaneous abortions is limited [23, 54]. A USA study [54] based on safety surveillance, with no control group, reported 41 miscarriages among 233 pregnancies exposed to duloxetine (17.6\%). Although surveillance studies are not ideal to establish prevalence, the rate of abortion was interpreted to be similar to background population (12-15\%). A Danish study [23] of pregnancies between 1997 
and 2008 found an increased risk of spontaneous abortions for women exposed to antidepressants, and included specific analyses of duloxetine exposure. However, the analyses were unadjusted, and the findings were attributed to confounding.

The association between duloxetine and elective abortions has not previously been reported in the available literature. The present study showed an increased hazard for duloxetine-exposed women when compared with duloxetine non-exposed, SSRI-exposed, and discontinuers. A lower and statistically non-significant hazard was seen when duloxetine-exposed women were compared with venlafaxineexposed women. Similar results were found in the sensitivity analyses. The decision is likely multifactorial if an elective abortion is chosen [30], with health issues related to the fetus among the factors. A recent US-based study did not find an increased risk for malformation after duloxetine exposure [55]. In the present study, the duration of pregnancies was similar across comparators, and most were very early in pregnancy. We find it unlikely that malformation could be identified this early and drive the observed increased risk of elective abortion. In Denmark, pregnant women are offered an ultrasound examination in week 20 to detect malformation. Furthermore, a similar hazard of elective abortions for venlafaxine and duloxetine-exposed women were found. The effect could be drug-class related; however, it could also be due to similarity in patients' underlying disease condition. Both venlafaxine and duloxetine are second-line treatments after SSRIs, therefore we believe that women taking these two drugs are likely to have similar depression severity [56] or similar health conditions that may have factored into their abortion decision.

\subsection{Strengths and Limitations}

The present study included a range of confounders, as opposed to previous studies using surveillance data or crude analyses. Residual confounding is, of course, a possibility. Diagnoses of co-morbidity were gathered from the patient register and given in relation to hospital contacts. Consequently, co-morbidity, including depression, is likely to be under-reported in the present study. On the other hand, we identified women with depression requiring hospitalization (8\% among duloxetine-exposed women). Improved information on indication for treatments, severity of depression, and co-morbidities would have strengthened the study further. Hence, various comparator groups were used. By using nationwide registers of high validity and completeness, the probability of sampling bias, allocation bias, and lost to follow-up bias (selection bias) was minimal. Early pregnancies were captured, with valid information about LMP. A validation study [41] showed that the positive predictive value of the diagnosis of spontaneous abortion is $97.4 \%$. However, underestimation is likely, since women may experience a miscarriage without recognizing it or without a hospital encounter. Under-reporting has been estimated to be $30 \%$ and is probably due to miscarriages early in pregnancy [57]. Misclassification in exposure is also a possibility. The sensitivity analyses of requiring two or more prescriptions were conducted to increase the likelihood of analyzing truly exposed women.

\section{Conclusion}

For spontaneous abortion, we conclude that there is no increased risk associated with exposure to duloxetine. After adjustment and PS-matching, confounding seems to be reduced, but the increased risk seen in the comparison with SSRI-exposed women is likely due to residual confounding. Increased hazards for elective abortion among duloxetineexposed women were found across comparators, except when compared with venlafaxine, which may also have been attributable to unmeasured confounding. The elective abortions were performed early in pregnancy and presumably not due to malformation, but the reason for an elective abortion is likely multifactorial, and a challenging outcome to interpret. Thus, no causality can be drawn from the study.

Supplementary Information The online version contains supplementary material available at https://doi.org/10.1007/s40801-021-00252-9.

Acknowledgements Thanks to Simone Møller Hede (ApHER) for her help coordinating the project, and her inputs to previous versions of the manuscript.

\section{Declarations}

Funding This publication is based on a post-authorization safety study (PASS) for duloxetine funded by Eli Lilly and Company, the manufacturer of duloxetine.

Conflicts of interest/competing interests The study was performed by the Copenhagen Phase IV Unit (Phase4CPH) at the Department of Clinical Pharmacology and the Institute of Applied Economics and Health Research Aps (ApHER), and financed by Eli Lilly and Company, the manufacturer of duloxetine. MZA, JP, and EJS have performed other studies regarding antidepressants involving funding from Janssen Pharmaceutical via their employment in Phase4CPH. TF is former employee at ApHER and currently employed by Quantify Research. JTA and TF have no relevant financial activities outside the submitted work. MFSF is a current employee of Eli Lilly and Company. HL and SPM are former employees of Eli Lilly and Company. HL is currently employed by Gilead Science Inc. and SPM is currently employed by Amgen Inc.

Ethics approval and consent to participate Due to the study's observational design using Danish registers, no ethics committee approval or consent from subjects were needed.

Consent for publication Not applicable. 
Availability of data and material The Danish national registers are protected by the Danish Act on Processing of Personal Data and can be accessed following an approved application. The researchers do not store any data and cannot hand over data.

Code availability Not applicable.

Authors' contributions All authors have participated sufficiently in the work to take public responsibility for the whole content. MZA, JP, JTA, HL, TF, and EJS contributed to the concept and design of the study. MZA, JP, JTA, HL, SPM, TF, and EJS contributed to the acquisition, analysis, and/or interpretation of data. MZA drafted the manuscript. JP, JTA, HL, SPM, TF, and EJS revised the manuscript. MZA and JP performed the statistical analysis. HL and SPM obtained funding.

Open Access This article is licensed under a Creative Commons Attribution-NonCommercial 4.0 International License, which permits any non-commercial use, sharing, adaptation, distribution and reproduction in any medium or format, as long as you give appropriate credit to the original author(s) and the source, provide a link to the Creative Commons licence, and indicate if changes were made. The images or other third party material in this article are included in the article's Creative Commons licence, unless indicated otherwise in a credit line to the material. If material is not included in the article's Creative Commons licence and your intended use is not permitted by statutory regulation or exceeds the permitted use, you will need to obtain permission directly from the copyright holder. To view a copy of this licence, visit http://creativecommons.org/licenses/by-nc/4.0/.

\section{References}

1. Evans J, Heron J, Francomb H, Oke S, Golding J. Cohort study of depressed mood during pregnancy and after childbirth. BMJ. 2001:257-60.

2. Gotlib IH, Whiffen VE, Mount JH, Milne K, Cordy NI. Prevalence rates and demographic characteristics associated with depression in pregnancy and the postpartum. J Consult Clin Psychol. 1989:269-74.

3. Bennett HA, Einarson A, Taddio A, Koren G, Einarson TR. Prevalence of depression during pregnancy: systematic review 1. Obstet Gynecol. 2004;103:698-709.

4. Chatillon O. Even C [Antepartum depression: Prevalence, diagnosis and treatment]. Encephale. 2010;36:443-51.

5. Kendell RE, Wainwright S, Hailey A, Shannon B. The influence of childbirth on psychiatric morbidity. Psychol Med. 1976:297-302.

6. Reefhuis J, Rasmussen SA, Friedman JM. Selective serotoninreuptake inhibitors and persistent pulmonary hypertension of the newborn. New Engl J Med. 2006;354:2188-90.

7. Cooper WO, Willy ME, Pont SJ, Ray WA. Increasing use of antidepressants in pregnancy. Am J Obstet Gynecol. 2007;196:544-5.

8. Andrade SE, Raebel MA, Brown J, Lane K, Livingston J, Boudreau $\mathrm{D}$, et al. Use of antidepressant medications during pregnancy: a multisite study. Am J Obstet Gynecol. 2008;198:194-5.

9. Jimenez-Solem E, Andersen JT, Petersen M, Broedbaek K, Andersen NL, Torp-Pedersen C, et al. Prevalence of antidepressant use during pregnancy in Denmark, a nation-wide cohort study. PLoS One. 2013. https://doi.org/10.1371/journal.pone. 0063034.

10. Sun Y, Dreier JW, Liu X, Ingstrup KG, Mægbæk ML, MunkOlsen T, et al. Trend of antidepressants before, during, and after pregnancy across two decades - a population-based study. Brain Behav. 2019. https://doi.org/10.1002/brb3.1441.
11. Kaufman DW, Kelly JP, Rosenberg L, Anderson TE, Mitchell AA. Recent patterns of medication use in the ambulatory adult population of the United States. The Slone Survey. JAMA. 2002:337-44.

12. Andrade SE, Gurwitz JH, Davis RL, Chan KA, Finkelstein JA, Fortman K, et al. Prescription drug use in pregnancy. Am J Obstet Gynecol. 2004;191:398-407.

13. Huybrechts KF, Palmsten K, Mogun H. National trends in antidepressant medication treatment among publicly insured pregnant women. Gen Hosp Psychiat. 2013:265-71.

14. Treatment guidelines including drug recommendation for medical treatment of unipolar depression [DANISH]. The Committee for the Medical Treatment of Unipolar Depression under the Council for the Use of Expensive Hospital Medicine; 2015.

15. Bassiouni BA, Rafei AA. 5-Hydroxytryptamine (serotonin), copper and ceruloplasmin plasma concentrations in spontaneous abortion. Eur J Obstet Gynecol R B. 1979;9:81-8.

16. Pastuszak A, Schick-Boschetto B, Zuber C, Feldkamp M, Pinelli M, Sihn S, et al. Pregnancy outcome following first-trimester exposure to fluoxetine (Prozac). JAMA. 1993;269:2246-8.

17. McElhatton PR, Garbis HM, Elefant E, Vial T, Bellemin B, Mastroiacovo $\mathrm{P}$, et al. The outcome of pregnancy in 689 women exposed to therapeutic doses of antidepressants. A collaborative study of the European Network of Teratology Information Services (ENTIS). Reprod Toxicol. 1996;10:285-94.

18. Chambers CD, Johnson KA, Dick LM, Felix RJ, Jones KL. Birth outcomes in pregnant women taking fluoxetine. New Engl J Med. 1996;335:1010-5.

19. Goldstein DJ, Corbin LA, Sundell KL. Effects of first-trimester fluoxetine exposure on the newborn. Obstet Gynecol. 1997;89:713-8.

20. Kulin NA, Pastuszak A, Sage SR, Schick-Boschetto B, Spivey G, Feldkamp M, et al. Pregnancy outcome following maternal use of the new selective serotonin reuptake inhibitors: a prospective controlled multicenter study. JAMA. 1998;279:609-10.

21. Sivojelezova A, Shuhaiber S, Sarkissian L, Einarson A, Koren G. Citalopram use in pregnancy: prospective comparative evaluation of pregnancy and fetal outcome. Am J Obstet Gynecol. 2005;193:2004-9.

22. Diav-Citrin O, Shechtman S, Weinbaum D, Wajnberg R, Avgil M, Di GE, et al. Paroxetine and fluoxetine in pregnancy: a prospective, multicentre, controlled, observational study. Br J Clin Pharmacol. 2008;66:695-705.

23. Kjaersgaard MIS, Parner ET, Vestergaard M, Sorensen MJ, Olsen J, Christensen J, et al. Prenatal antidepressant exposure and risk of spontaneous abortion - a population-based study. PLoS One. 2013. https://doi.org/10.1371/journal.pone.0072095.

24. Andersen JT, Andersen NL, Horwitz H, Poulsen HE, JimenezSolem E. Exposure to selective serotonin reuptake inhibitors in early pregnancy and the risk of miscarriage. Obstet Gynecol. 2014;124:655-61.

25. Zuckerman B, Amaro H, Bauchner H, Cabral H. Depressive symptoms during pregnancy: relationship to poor health behaviors. Am J Obstet Gynecol. 1989:1107-11.

26. Smedberg J, Lupattelli A, Mardby AC, Overland S, Nordeng H. The relationship between maternal depression and smoking cessation during pregnancy-a cross-sectional study of pregnant women from 15 European countries. Arch Womens Ment Health. 2015:73-84

27. Richardson JL, Martin F, Dunstan H, Greenall A, Stephens S, Yates LM, et al. Pregnancy outcomes following maternal venlafaxine use: a prospective observational comparative cohort study. Reprod Toxicol. 2019;84:108-13.

28. Almeida ND, Basso O, Abrahamowicz M, Gagnon R, Tamblyn $\mathrm{R}$. Risk of miscarriage in women receiving antidepressants in early pregnancy. Correcting for induced abortions. Epidemiology. 2016;27:538-46. 
29. Kieler H, Malm H, Artama M, Engeland A, Furu K, Gissler $\mathrm{M}$, et al. Use of antidepressants and association with elective termination of pregnancy: population based case-control study. BJOG. 2015;122:1618-24.

30. Biggs MA, Gould H, Foster DG. Understanding why women seek abortions in the US. BMC Womens Health. 2013;13:29.

31. Kildemoes HW, Sorensen HT, Hallas J. The Danish National Prescription Registry. Scand J Public Health. 2011;39:38-41.

32. Gaist D, Sorensen HT, Hallas J. The Danish prescription registries. Dan Med Bull. 1997;44:445-8.

33. Schmidt M, Schmidt SAJ, Sandegaard JL, Ehrenstein V, Pedersen L, Sørensen HT. The Danish National Patient Registry: a review of content, data quality, and research potential. Clin Epidemiol. 2015;7:449-90.

34. Kristensen J, Langhoff-Roos J, Skovgaard LT, Kristensen FB. Validation of the Danish Birth Registration. J Clin Epidemiol. 1996;49:893-7.

35. Langhoff-Roos J, Krebs L, Klungsøyr K, Bjarnadottir RI, Källén $\mathrm{K}$, Tapper A-M, et al. The Nordic medical birth registers-a potential goldmine for clinical research. Acta Obstet Gynecol Scan. 2014;93:132-7.

36. Baadsgaard M, Quitzau J. Danish registers on personal income and transfer payments. Scand J Public Health. 2011;39:103-5.

37. Jensen VM, Rasmussen AW. Danish education registers. Scand J Public Health. 2011;39:91-4.

38. DENMARK. Law No. 350 of 13 June 1973 on the interruption of pregnancy. https://cyber.harvard.edu/population/abortion/ Denmark.abo.htm. Accessed 20 June 2019.

39. Abortion in Denmark. Wikipedia. 2019. https://en.wikipedia. org/w/index.php?title=Abortion_in_Denmark\&oldid=89551 6682. Accessed 20 June 2019.

40. Källén B, Olausson PO. No increased risk of infant hypospadias after maternal use of loratadine in early pregnancy. Int $\mathrm{J}$ Med Sci. 2006;3:106-7.

41. Lohse SR, Farkas DK, Lohse N, Skouby SO, Nielsen FE, Lash TL, et al. Validation of spontaneous abortion diagnoses in the Danish National Registry of Patients. Clin Epidemiol. 2010;2:247-50.

42. Larsen H, Nielsen GL, Bendsen J, Flint C, Olsen J, Sorensen HT. Predictive value and completeness of the registration of congenital abnormalities in three Danish population-based registries. Scand J Public Health. 2003;31:12-6.

43. Knudsen LB, Olsen J. The Danish Medical Birth Registry. Dan Med Bull. 1998;45:320-3.

44. Parsons LS. Performing a $1: \mathrm{N}$ case-control match on propensity score. SUGI 29. Montréal, Canada; 2004. p. Paper 165-29.
45. Yang D, Dalton JE. A unified approach to measuring the effect size between two groups using SAS. SAS global forum, Paper 335-2012. 2012. https://support.sas.com/resources/papers/proce edings12/335-2012.pdf. Accessed 11 Nov 2020.

46. Dalton JE. A new standardized difference metric for multinomial samples. Unpublished work. 2008;

47. World Health Organisation. Defined daily dose (DDD). http:// www.who.int/medicines/regulation/medicines-safety/toolkit_ddd/ en/. Accessed 29 Apr 2020.

48. Jacob L, Gerhard C, Kostev K, Kalder M. Association between induced abortion, spontaneous abortion, and infertility respectively and the risk of psychiatric disorders in 57,770 women followed in gynecological practices in Germany. J Affect Disord. 2019;251:107-13.

49. Gillespie SL, Mitchell AM, Kowalsky JM, Christian LM. Maternal parity and perinatal cortisol adaptation: the role of pregnancyspecific distress and implications for postpartum mood. Psychoneuroendocrinology. 2018;97:86-93.

50. Cohain JS, Buxbaum RE, Mankuta D. Spontaneous first trimester miscarriage rates per woman among parous women with 1 or more pregnancies of 24 weeks or more. BMC Preg Childb. 2017; $17: 437$.

51. Andersen A-MN, Andersen PK, Olsen J, Grønbæk M, StrandbergLarsen K. Moderate alcohol intake during pregnancy and risk of fetal death. Int J Epidemiol. 2012;41:405-13.

52. Baba S, Noda H, Nakayama M, Waguri M, Mitsuda N, Iso H. Risk factors of early spontaneous abortions among Japanese: a matched case-control study. Hum Reprod. 2011;26:466-72.

53. George L, Mills JL, Johansson ALV, Nordmark A, Olander B, Granath F, et al. Plasma folate levels and risk of spontaneous abortion. JAMA. 2002;288:1867-73.

54. Hoog SL, Cheng Y, Elpers J, Dowsett SA. Duloxetine and pregnancy outcomes: safety surveillance findings. Int J Med Sci. 2013:413-9.

55. Huybrechts KF, Bateman BT, Pawar A, Bessette LG, Mogun H, Levin R, et al. Maternal and fetal outcomes following exposure to duloxetine in pregnancy: cohort study. BMJ. 2020;368:m237.

56. Danish Psychiatric Society: Treatment with psychotropics [DANISH] http://dpsnet.dk/wp-content/uploads/2014/12/anvendelse_ af_psykofarmaka_okt_2014.pdf. Accessed 18 Jul 2019.

57. Buss L, Tolstrup J, Munk C, Bergholt T, Ottesen B, Grønbaek $\mathrm{M}$, et al. Spontaneous abortion: a prospective cohort study of younger women from the general population in Denmark. Validation, occurrence and risk determinants. Acta Obstet Gynecol Scan. 2006;85:467-75

\section{Authors and Affiliations}

\section{Mikkel Zöllner Ankarfeldt ${ }^{1,7}$ (1) - Janne Petersen ${ }^{1,2}$ • Jon Trærup Andersen ${ }^{3,4}$. Maria Fernanda Scantamburlo Fernandes ${ }^{5} \cdot \mathrm{Hu} \mathrm{Li}^{5} \cdot$ Stephen Paul Motsko ${ }^{5} \cdot$ Thomas Fast $^{6} \cdot$ Espen Jimenez-Solem $^{1,3,4}$}

1 Copenhagen Phase IV Unit (Phase4CPH), Department of Clinical Pharmacology and Center for Clinical Research and Prevention, Copenhagen University Hospital Bispebjerg and Frederiksberg, Copenhagen, Denmark

2 Section for Biostatistics, Department of Public Health, University of Copenhagen, Copenhagen, Denmark

3 Department of Clinical Pharmacology, Copenhagen University Hospital Bispebjerg and Frederiksberg, Copenhagen, Denmark
4 Faculty of Health and Medical Sciences, University of Copenhagen, Copenhagen, Denmark

5 Eli Lilly and Company, Indianapolis, IN, USA

6 Institute of Applied Economics and Health Research, Copenhagen, Denmark

7 Center for Clinical Research and Prevention, Frederiksberg Hospital, Hovedvejen Indgang 5, Nordre Fasanvej 57, 2000 Frederiksberg, Denmark 